\title{
Malignant Melanoma within a Cellular Blue Nevi Presenting as a Vascular Malformation and the Connection to Sporadic KRAS Mutations
}

\author{
Felicia Tai ${ }^{a} \quad$ Vitor M. Pereirab ${ }^{b}$ Sam Babak $^{c}$ Ivan Radovanovic ${ }^{d}$ \\ Shachar Sade ${ }^{e}$ Tara Lynn Teshima ${ }^{f}$ \\ aTemerty Faculty of Medicine, University of Toronto, Toronto, ON, Canada; ${ }^{\circ}$ Division \\ of Neurosurgery, Department of Surgery, University of Toronto, Toronto, ON, Canada; \\ 'Medical Oncology, Markham Stouffville Hospital, Markham, ON, Canada; dDivision of \\ Neurosurgery, Department of Surgery, Toronto Western Hospital, Toronto, ON, Canada; \\ eDepartment of Pathology, Sunnybrook Health Sciences Centre, Toronto, ON, Canada; \\ fDivision of Plastic and Reconstructive Surgery, Department of Surgery, Markham Stouffville \\ Hospital, Markham, ON, Canada
}

Keywords

Melanoma $\cdot$ Vascular malformation $\cdot$ Blue nevus $\cdot$ Nevus $\cdot$ KRAS

\section{Abstract}

We present a case of malignant melanoma (MM) developing within a vascular malformation showing features of cellular blue nevi. A 47-year-old male presented with acute symptoms of a temporal and zygomatic mass, which were both previously asymptomatic upon development 30 years ago. These masses were diagnosed as vascular malformations upon imaging and were treated with sclerotherapy. Embolization and surgical excision were performed 3 years later due to symptomatic growth. Final pathology reports showed MM with congenital blue nevi. We hypothesize a possible linkage to a sporadic KRAS mutation, linking both presentations of vascular malformation, MM, and cellular blue nevi. A literature search for similar cases is also reported.

\section{Introduction}

Malignant melanoma (MM) is one of the most aggressive skin cancers globally. In Canada, rates of MM have been steadily increasing since 1984 and account for $\sim 1.5 \%$ of all cancer deaths [1]. Classic risk factors may include fair skin pigmentation, UV radiation exposure, age, 
and immunosuppression. However, high total body nevus counts, dysplastic nevi, and congenital nevi such as cellular blue nevi may also correlate with malignant transformation of skin lesions.

While blue nevi are benign melanocytic lesions, "de novo" MM lesions may derive from blue nevi lesions, indicating then a potential correlation between the 2 entities. Furthermore, it is known that $70 \%$ of melanomas may arise de novo in the skin, while only $30 \%$ arise within a preexisting nevus or a congenital nevus [2]. In large congenital melanocytic nevi, the risk of developing cutaneous or extracutaneous melanoma is $2-5 \%$ over the lifetime, with most melanomas occurring in the first 5 years of life [3].

Vascular malformations are congenital lesions that can be composed of veins, arteries, lymphatic tissue, or a combination of these tissues. Each malformation type may require a different management strategy to mitigate the risk of bleeding, mass effect, or other symptoms like pain or swelling. Slow-flow lesions such as venous malformations the most common ones and they are mainly treated with sclerotherapy or laser therapy, while the mainstay for capillary malformations is laser therapy. Arteriovenous malformations are fast-flow lesions and a complex management that may include embolization with or without surgical excision.

There are currently 7 cases reported in the literature of MM mimicking or presenting within various types of vascular malformations, indicating a possible link in development [4-9]. We present a rare case of a 47-year-old male diagnosed with a subcutaneous MM arising from a congenital blue nevus presenting as a venous malformation.

\section{Case Report}

A male patient presented with a mass over his right zygomatic region in his $20 \mathrm{~s}$. X-ray at the time diagnosed the mass as a "bone growth" and no further treatments were sought. Over 20 years, the mass grew slowly documented in photos and through patient recollection. Asides from reporting migraines with aura occurring since high school, the patient did not experience any other symptoms. A second mass over the right temporal muscle appeared around age 40 and began slowly progressing in size.

At age 47, the patient experienced an insect bite directly on the right temporal mass while travelling in Southern India. Upon returning to Canada 3 days later, he presented to the emergency department with fever, pain, and swelling in the right temporal area where the mass was located. Antibiotics were used successfully to treat constitutional symptoms over 2 weeks. A CT scan confirmed a mass overlying his right temporal muscle measuring $2.4 \times 1.7$ $\times 2.0 \mathrm{~cm}$ and a second mass overlying his right zygoma measuring $1.9 \times 3.7 \times 3.9 \mathrm{~cm}$. Further investigation with magnetic resonance imaging (MRI) with gadolinium demonstrates a vascular pattern correspondent to a venous malformation. Scalloping of the right zygoma was also present, consistent with a chronic lesion. Five sessions of bleomycin sclerotherapy were performed over 3 years after the presumptive diagnosis of a venous malformation (Fig. 1a). Surgical options were discussed however, the patient declined.

Two years later, the patient was referred to the senior authors V.P. and T.L.T for definitive treatment (Fig. 1b). An updated MRI showed the zygoma mass measuring $2.1 \times 3.9 \times 4.5 \mathrm{~cm}$, and the temporalis mass measuring $5.8 \times 1.9 \times 6.0 \mathrm{~cm}$, both larger in size than the baseline CT prior to bleomycin treatment (Fig. 1c). Percutaneous glue embolization of both masses was performed followed by immediate surgical excision. Following surgical excision, the patient no longer experienced migraines with aura. The pathology results reported the right zygoma mass as a nodular spindle cell lesion with fat necrosis. A second and third opinion reported the zygoma mass as a deeply infiltrating blue nevus with congenital features. Immunohistological staining was positive for S-100, HMB45, and CD56 and negative for CD34,

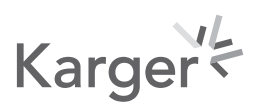



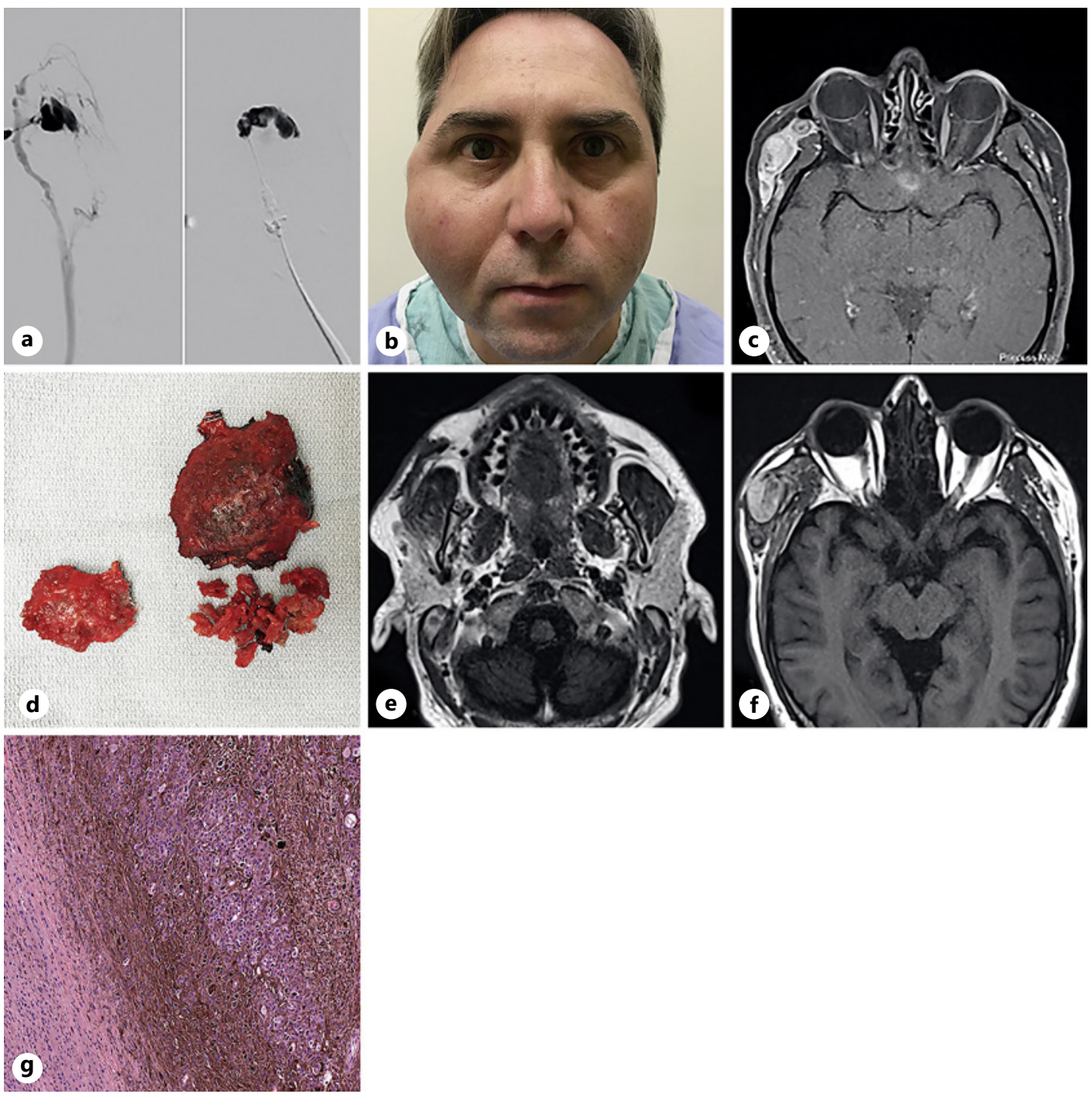

Fig. 1. a Direct venogram during bleomycin injection and DSA showing dilated venous pouches and a delayed venous drainage. $\mathbf{b}$ Image of patient at the time of the initial consultation at Toronto Vascular Malformation Clinic. c Axial MRI T1 fat sat - right temporal gadolinium-enhanced nodular lesion with heterogenous signal suggesting a vascular malformation type venous with partial thrombosed venous channels. $\mathbf{d}$ Intraoperative cheek and temporal mass. e Axial MRI T1 images of right-side surgical scar with complete resection of the masseterian located venous malformation. $\mathbf{f}$ Temporal nodular heterogenous lesion suggestive of a tumor. g Histological section of right temporal mass (×200 magnification) showing transition from a background blue nevus (left side) to melanoma showing an expansile nodular proliferation of enlarged atypical melanocytes (right side). MRI, magnetic resonance imaging; DSA, digital subtracted angiogram.

CD31, AE1, and desmin. All 3 pathologists reported the right temporal mass as adipose tissue with fat necrosis.

Six months after the surgical excision, the patient presented with a recurrent mass in the right temporal area growing rapidly over the course of 1 month. MRI showed a dilated venous varix measuring $6.6 \times 2.8 \times 7.0 \mathrm{~cm}$ (Fig. 1f). Sclerotherapy was not performed prior to repeat surgery as a venous pouch could not be found on ultrasound. Subsequent surgical excision was complicated by excessive blood loss, characteristic of a venous malformation.

New histopathology reported a final diagnosis of MM arising intradermally within a preexisting cellular blue nevus with atypia (Fig. 1g). The surgical specimen contained a 
cellular spindled melanocytic lesion with some admixed melanin pigment. The appearance was consistent with a cellular blue nevus, with some mild cellular pleomorphism and focal cytologic atypia (i.e., compatible with "atypical cellular blue nevus"). Within the lesion, there was also an expansile nodular lesion ( $2.5 \mathrm{~cm}$ in maximal dimension) showing confluent sheetlike growth of enlarged atypical epithelioid melanocytes with scattered mitoses consistent with transformation to MM. The expansile sheet-like growth of enlarged epithelioid cells showing cytologic atypia and mitotic activity within the nodular lesion was considered to be consistent with transformation to melanoma, as opposed to the possibility of a "satellite" nodule within a blue nevus.

Once a firm diagnosis of MM was confirmed after extensive pathology review of all specimens, a thorough full-body skin exam was performed without identification of a possible alternative primary. Further staging investigations including CT head and neck, thorax, abdomen, and pelvis; PET/CT and brain MRI did not show any evidence of metastasis. The patient had a negative family history of melanoma and other cancers. Next-generation sequencing for melanoma panel was negative, including BRAF mutations. The patient was started on adjuvant nivolumab for 6 months but discontinued due to recurrent grade 2 lichen planus.

\section{Discussion}

To our knowledge, this is the first report of MM related to a venous malformation and cellular blue nevi. Currently in the literature, there are 7 reports of MM isolated from various types of vascular malformations (Table 1). 2/7 of the cases were MM developing within a vascular malformation $[4,5]$, while $5 / 7$ of cases were MM mimicking a vascular malformation located in the orbit [6] or intracranially [7, 9]. Testicular choriocarcinoma and small-cell lung cancer have also been reported to be found developing within arteriovenous malformations $[8,10]$. Similar to our presented case, lesions were treated as a vascular malformation until the diagnosis of melanoma on histopathological examination following surgical resection. We postulate this may be due to the 2 entities coexisted and the imaging diagnosis of MM misguided by the appearance of the vascular lesions.

Most similar to our case, a 75-year-old patient had a MM growing within a capillary malformation, which developed from a nonpigmented birthmark on his back [5]. However, there was no association of this case to cellular blue nevi. Lee et al. [6] reported 2 cases of primary orbital melanoma mimicking vascular malformations. Three cases of MM presented as intracranial hemorrhages of vascular malformations [7, 9]. El-Sawy et al. [11] describe 3 cases of primary orbital melanoma with cellular blue nevus features. The first case was transformed from a congenital blue nevi birthmark. However, like our case, the other 2 cases of primary orbital melanoma did not present with cutaneous pigmentation, and only showed cellular blue nevus features under pathological inspection [11]. The latter 2 cases also did not have any other sites of disease involvement upon inspection. Hussain et al. [12] report a case of slow-growing MM developing over 40 years from an orbital cellular blue nevi with no cutaneous presentation.

Recently, somatic mutations in the KRAS gene pathway have been described in association with superficial or brain AVM malformations. KRAS is a proto-oncogene involved in cell signaling of the RAS/MAPK signaling pathway and is coded by the KRAS gene. Mutations in the $K R A S$ gene, an upstream regulator of $B R A F$ signaling, have been reported in blue nevi [13], vascular malformations [14], and MM. Emley et al. [13] found 22\% cases of cellular blue nevi had KRAS mutations. Nikolaev et al. [14] found KRAS mutations in up to $62.5 \%$ of arteriovenous malformations tissue samples. In MM, KRAS mutations were reported in up to $2 \%$ of

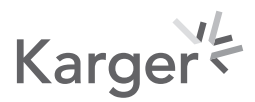




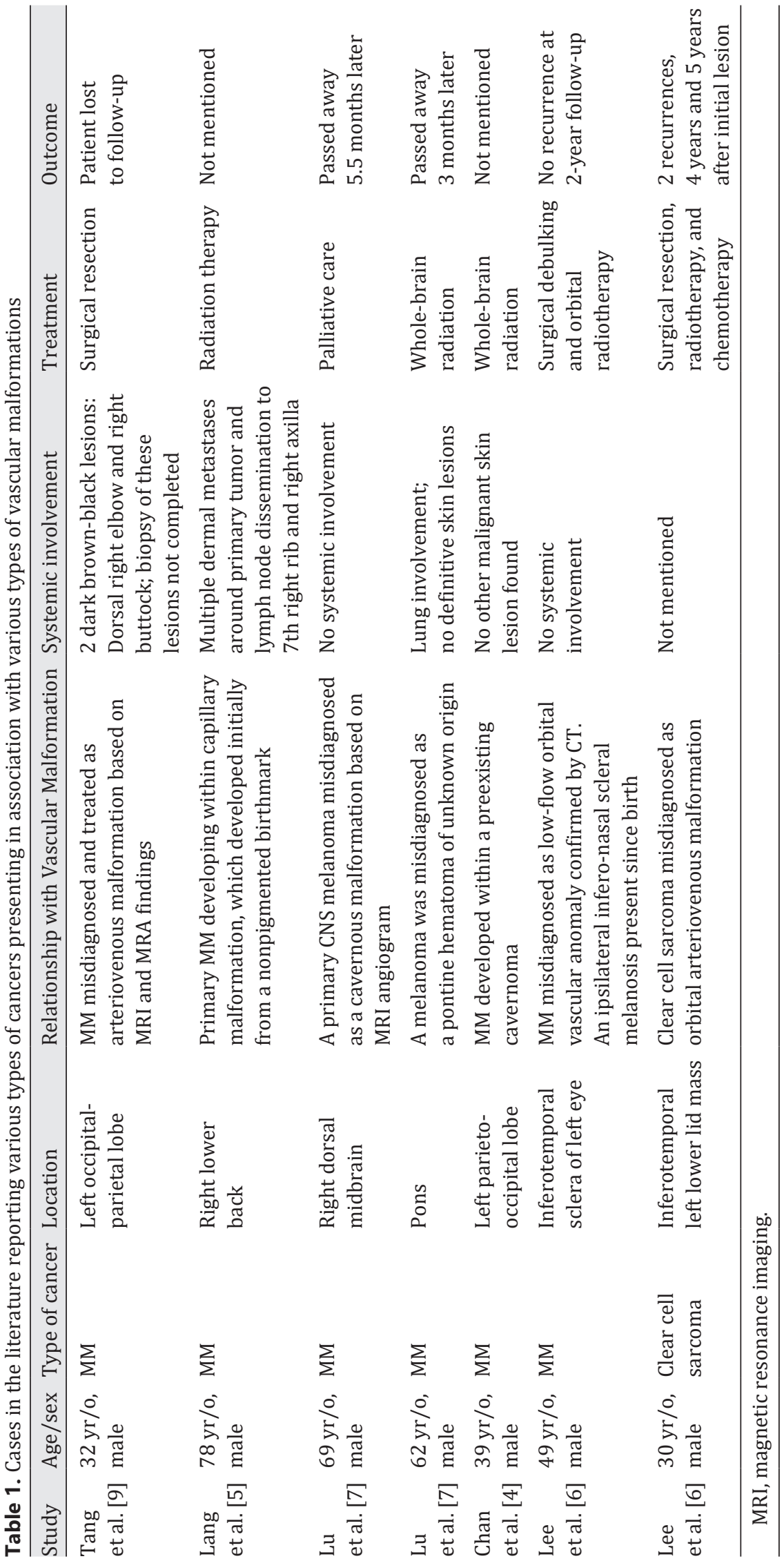


cases [15] and were found to be integral for melanoma development in a mouse model [16]. Thus, we speculate that a sporadic KRAS mutation early on may have led to development of both a vascular malformation-like presentation and blue nevi which transformed over decades into a MM. This correlates with the negative finding of BRAF mutations, which is downstream to KRAS signaling.

\section{Conclusion}

Ultimately, this case exhibits a unique presentation of MM in association with congenital blue nevi and a venous malformation. The presence of both a vascular malformation and MM in a localized mass led to a grey area regarding diagnosis and management. Our case in conjunction to the cases reported in literature proposes a possible linkage between MM within vascular malformations through possibly somatic KRAS gene mutations.

\section{Statement of Ethics}

The patient provided written informed consent to the writing of this article and the publication of pictures. The research was conducted ethically in accordance with the World Medical Association Declaration of Helsinki.

\section{Conflict of Interest Statement}

The authors have no conflicts of interest to declare.

\section{Funding Sources}

No sources of funding were required in the preparation of this manuscript.

\section{Author Contributions}

Authors T.L.T. and F.T. made substantial contributions to conception and design of the work. Authors T.L.T., V.P., S.B., I.R., and S.S. made substantial contributions to the acquisition, analysis, and interpretation of data. All the authors (F.T., V.P., S.B., I.R., S.S., and T.L.T.) were involved in drafting or revising of the work for important intellectual content, final approval of version to be published, and agree to be accountable for all aspects of the work.

\section{References}

1 Committee CCSA. Canadian cancer statistics 2019. Toronto, ON: Canadian Cancer Society; 2019.

2 Longo C, Rito C, Beretti F, Cesinaro AM, Piñeiro-Maceira J, Seidenari S, et al. De novo melanoma and melanoma arising from pre-existing nevus: in vivo morphologic differences as evaluated by confocal microscopy. J Am Acad Dermatol 2011;65(3):604-14.

3 Vourc'h-Jourdain M, Martin L, Barbarot S. Large congenital melanocytic nevi: therapeutic management and melanoma risk: a systematic review. J Am Acad Dermatol. 2013;68(3):493-14. e491414

4 Chan CH, Fabinyi GC, Kalnins RM. An unusual case of tumor-to-cavernoma metastasis. A case report and literature review. Surg Neurol. 2006;65(4):402-9. 
5 Lang CL, Øregaard JS, Paulsen JF, Jemec B. Unique presentation of a malignant melanoma in a capillary malformation: a case report. JPRAS Open 2015;5:4-6.

6 Lee V, Sandy C, Rose GE, Moseley IM, Cree I, Hungerford JL. Primary orbital melanoma masquerading as vascular anomalies. Eye. 2002;16(1):16-20.

7 Lu AY, Patel AR, Kuzmik GA, Atsina KK, Bronen RA, Jabbour PM, et al. Brainstem melanomas presenting as a cavernous malformation. Neurochirurgie 2014;60(4):184-7.

8 Sundarakumar DK, Marshall DA, Keene CD, Rockhill JK, Margolin KA, Kim LJ. Hemorrhagic collision metastasis in a cerebral arteriovenous malformation. BMJ Case Rep. 2014.

9 Tang Y, Hou Y, Prasad B, Yang W, Wang J. Intracerebral malignant melanoma presenting as an Arteriovenous Malformation: case report and literature review. Chin Neurosurg. 2017;3(1):15.

10 Trapella G, Migliore MM, Roccella P, Cavazzini L. Metastatic carcinoma to artero-venous haemangioma. Case report. J Neurosurg Sci 1993;37(1):35-8.

11 El-Sawy T, Bakhoum MF, Tetzlaff M, Nasser QJ, Prieto VG, Ivan D, et al. Primary orbital melanoma in association with cellular blue nevus. Digit J Ophthalmol. 2014;20(3):35-40.

12 Hussain A, Sidiropoulos M, Das S, Munoz DG, Nijhawan N. Orbital cellular blue nevus complicated by malignant melanoma. Can J Ophthalmol. 2017;52(3):e111-3.

13 Emley A, Nguyen LP, Yang S, Mahalingam M. Somatic mutations in GNAQ in amelanotic/hypomelanotic blue nevi. Hum Pathol 2011;42(1):136-40.

14 Nikolaev SI, Vetiska S, Bonilla X, Boudreau E, Jauhiainen S, Rezai Jahromi B, et al. Somatic activating KRAS mutations in arteriovenous malformations of the brain. N Engl J Med 2018;378(3):250-61.

15 Cicenas J, Tamosaitis L, Kvederaviciute K, Tarvydas R, Staniute G, Kalyan K, et al. KRAS, NRAS and BRAF mutations in colorectal cancer and melanoma. Med Oncol. 2017;34(2):26.

16 Milagre C, Dhomen N, Geyer FC, Hayward R, Lambros M, Reis-Filho JS, et al. A mouse model of melanoma driven by oncogenic KRAS. Cancer Res 2010;70(13):5549-57. 